\title{
Density Based Traffic Controller with Emergency Override using CCTV Camera
}

\author{
Venkat G, Arulananth T S, Baskar M
}

\begin{abstract}
The aim of this project is to provide alternative solution for the traffic signal system in clearing high density traffic jam. Now a day's more number of vehicles is coming on to the road creating more traffic congestion at any junction. The traffic congestion is a severe problem when there arises high density at a particular junction. Especially when there is an emergency like ambulance, fire brigade stuck in the traffic they require priority to go first. In such cases it is necessary to override the normal signal timings automatically. To overcome this problem, this project uses CCTV cameras on each side of junction. It assigns longer green light with the help of the micro controller whenever it sensing the heavy density and whenever it finds emergency vehicles stuck in traffic like ambulance, fire brigade etc they require priority to go first. In this manner it overrides the standard signal timings there by it saves the waiting time of the vehicular. This project uses micro controller interfacing with CCTV aligned in the sight configuration across the load for detecting the density. Once the image is captured from the CCTV footage, it is converted into greyscale image. The greyscale image is passed through the median filter in order to reduce the noise present in it. Further Canny edge detection finds the intensity gradients of the images by suppressing all the other edges that weak and not connected to the strong edges. Then based on the canny image the density is calculated and turns the green light on at the heavy density road side of junction.
\end{abstract}

Keywords - traffic patterns, traffic density, Image acquisition, Canny edge detection, Median filter

\section{INTRODUCTION}

With the increasing in population, the traffic density on road has reached to an unprecedented level. Heavy traffic density leads to wastage of time, which increases the traffic congestion. Traffic control has become a major problem in developed metropolitan cities and towns. The traffic patterns are dynamic and constantly changing. It became more difficulty to clear the dynamic traffic. In such scenario, human intervention became pivotal role in clearing the traffic. Due to standard fixed signal timings there is formation of huge traffic on roads. The best solution to solve the traffic is to implement real time traffic control signal based on the road condition whether it is occupied with more traffic or not. This can be implemented through CCTV cameras that are interfaced with micro controller. The CCTV cameras input the captured images to the micro controller with the help of image processing techniques and filters. Based on the sensing traffic density the micro

Revised Manuscript Received on July 10, 2019.

Venkat G, PG student, Department of Electronics and Communication Engineering, MLR Institute of Technology, Hyderabad-43, Telangana, India. (E-mail: gvenkat1236@gmail.com)

Arulananth T S, Professor, Department of Electronics and Communication Engineering, MLR Institute of Technology, Hyderabad-43, Telangana, India. (E-mail: arulanandh.ts@gmail.com)

Baskar M, Professor, Department of Computer Science and Engineering, MLR Institute of Technology, Hyderabad, Telangana, India. (E-mail: baashkarcse@gmail.com) controller outputs the green and red signal lights. This system controls the traffic light duration i.e traffic light timer depending upon the road occupation. It also controls the traffic pass permission based on road related factors such as order, load, priority and wait time.

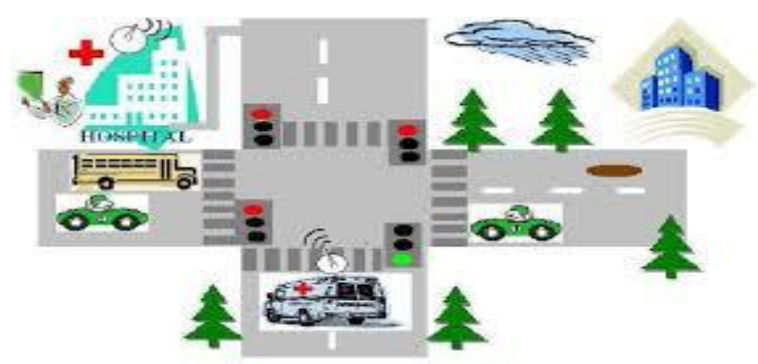

Fig.1. Traditional Traffic signal system

\section{EXISTING SYSTEM}

The existing traffic signals were fixed timed and irrespective of the volume. Vehicles had to wait for the signals to turn the green light on as per the pre-fixed timings. This same procedure repeating continuously irrespective of the traffic jam. At present all traffic junctions have built in priority rule, which means that heavy traffic in one direction gets minimum time and tend to dominate. It causes excessive delays to traffic in other directions.

Due to this built in standard allotted signal timings on each side of the road creating more waiting time to other sides of the vehicular. And it causes heavy traffic congestion when there is an emergency vehicles stuck in the junction.

To overcome these problems, a proposed system introduces new methodology that uses CCTV which is interfaced with micro controller. On sensing the traffic density, the micro controller overrides the normal signal timings automatically, without human intervention.

\section{PROPOSED METHODOLOGY}

The proposed methodology uses high definition CCTV cameras, motion sensors, buzzers will be installed at traffic junction. These high definition cameras, motion sensors will detects vehicle queues and turn the green light on to regulate the heavy density traffic flow. The cameras will monitor traffic coming from all directions. These smart traffic signals are unmanned and will allow movement depending on the number of the vehicles or traffic density on a given road at a given time. So, if vehicles on one of the roads passing through a crossing, it blows red light signal to other vehicles on the road.

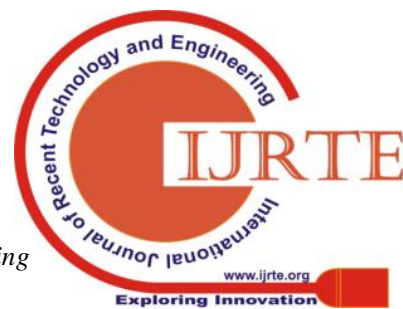


The other roads passing through a crossing will be cleared only after passing of high density traffic jam. This will save the time for commuters and reduces workload on the traffic cops.

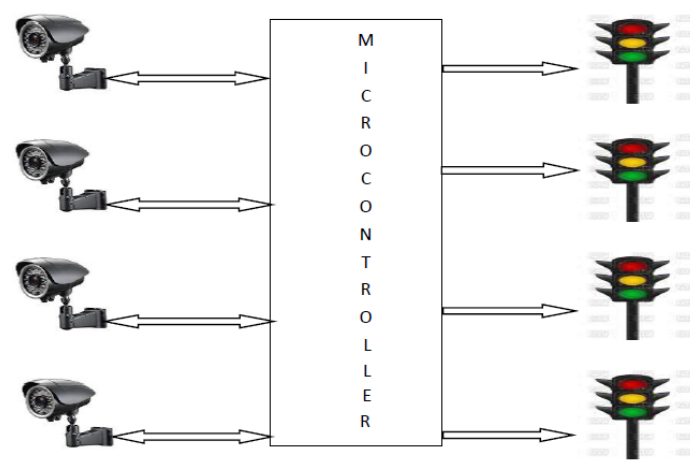

Fig.2. Block diagram of proposed method

In this method the CCTV cameras are always busy in capturing the traffic on each road side. These images are being fed to micro controller which gives commands to the traffic signals to turn green and red signals on automatically. Before being inputting the captured images to micro controller, they are filtered and undergo through some image processing techniques in order to reduce storage and noise. The following image processing steps are performed on traffic captured images before being fed to the micro controller.

Image acquisition: It is an image obtained by the cameras which contains colored image along with all other objects like buildings, trees etc. surrounded by the traffic scale image which is black and white with brightness.

Median filter: It is a non linear digital filter can remove noise from images which is typical step to improve the results of the later processing.

Threshold level: It is the process of transforming gray scale image into black and white by assigning binary 1 to white and binary 0 to black. It can reduce the redundancy information in order to simplify for further processing.

Canny edge detection: It is a multistage algorithm that is susceptible to noise in the image, finds intensity gradients of images and finalizes the edge detection by suppressing the all the other edges that are weak and not connected to strong edges without disturbing the original data in the image.

The resultant canny edge image is applied to the micro controller, based on this image it can estimates the traffic density and then it sends the commands to the respective traffic signals to turn the green, red signals.

\section{Algorithm:}

Step1: Capturing the traffic images on each road side of the junction.

Step2: Converting the captured images into grey scale images and then performing the image enhancement. The image enhancement improves the brightness of the images.

Step3: Filtering the enhanced traffic image with the non linear digital median filter that reduces the noise, interference present in the image without disturbing the original information. junction. All these images are being converted into grey

Step4: Threshold operation is performed on the filtered image by assigning binary digit 1 to white i.e. foreground and binary digit 0 to the black i.e. background in order to remove the unnecessary information which is not assigned either by binary digit 1 or by binary digit 0 in the grey scale image.

Step5: The resultant image is processed by the canny edge detection in which the intensity of the gradients and edges of the image are finalized.

Step6: The micro controller compares the traffic densities of all road sides in the following manner, if the cameral traffic density is greater than camera2, then it is again compared with cameras 3,4 respectively. If it found that camera1 traffic density is heavier than cameras3, 4 respectively then the micro controller turns the green light on camera1 direction, red light for other road sides.

If the camera2 traffic density is greater than camera3, then it is again compared with cameras 1,4 respectively. If it found that camera2 traffic density is heavier than cameras1, 4 respectively then the micro controller turns the green light on camera 2 direction, red light for other road sides.

If the camera3 traffic density is greater than camera4, then it is again compared with cameras1, 2 respectively. If it found that camera3 traffic density is heavier than cameras1,2 respectively then the micro controller turns the green light on cameral direction, red light for other road sides.

Step7: The above step 6 is continuously runs throughout the day.

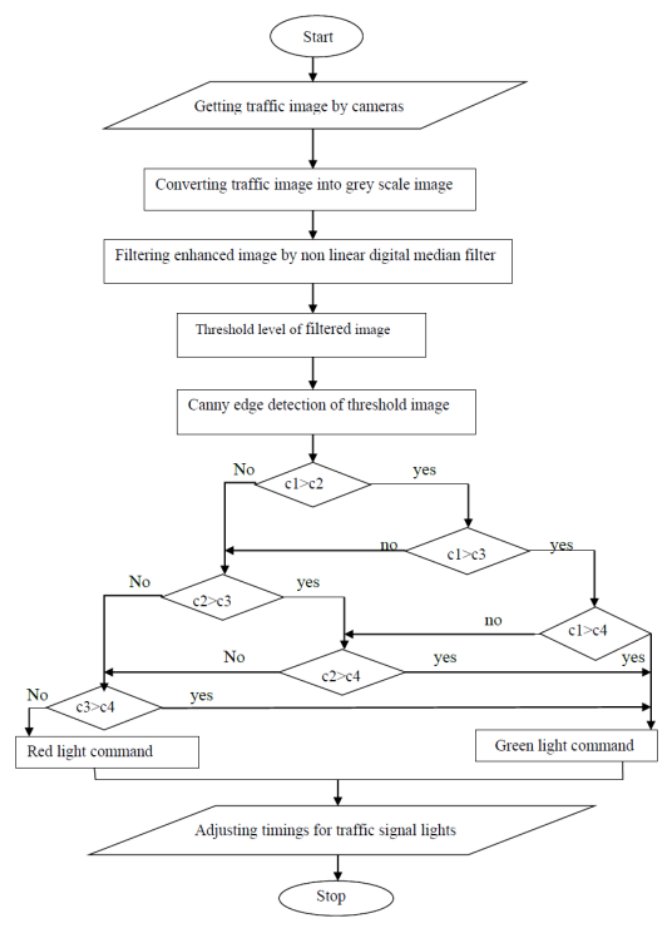

Fig3: Flow chart of controlling traffic signal 


\section{RESULTS AND DISCUSSION}

The results include the acquired images from the cameras that are fetched to the micro controller after processed by digital image processing techniques. These images are filtered with median filters. Once filtering operations performed on these images they are enhanced, threshold levels of each image will be estimated. Then the resultant canny edge detected image is applied to the micro controller which turns the traffic signals on by adjusting the timings. The resultant images are shown below.

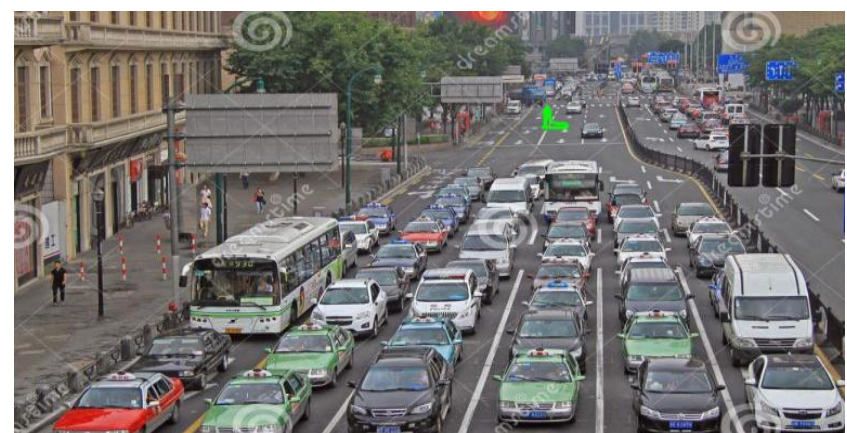

Fig.4. the captured traffic image by cameras at the junction

\section{Image Acquisition:}

The captured images from all road sides comprise all the vehicles, trees, hoardings, sign boards and footpath etc as shown in the figure 4 . These images occupy a lot of storage area. But here we need only the number of vehicles occupied the roads and other remaining area is to be neglected. For the purpose of obtaining only the number stuck in the traffic, the acquired images are to be converted into gray scale images and to be enhanced. The gray scale images are black and white images. It is main aspect of images where the brightness plays a pivotal role. It does not contain any coloured information of the image.

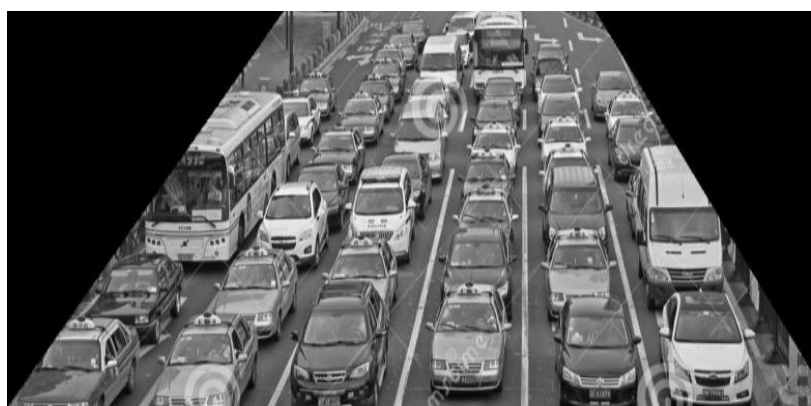

Fig.5. the filtered traffic image by removing unwanted information.

The resultant image is then filtered with a non linear digital median filter. It removes all unwanted component present in the image, thereby it reduces the noise. The filtering operation removes all other images like trees, sign boards, buildings, footpath area etc as shown in the figure 5 .

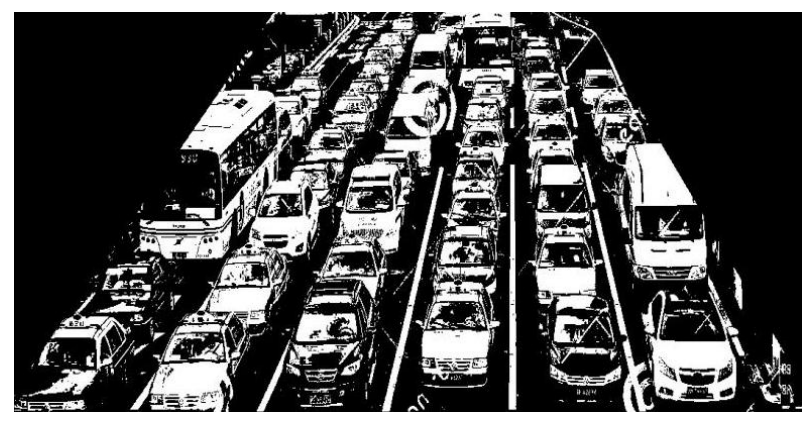

Fig.6. the threshold levels of traffic image

The filtered gray scale images are applied to the threshold process where all grey scale images are converted into binary images. In other words threshold is a non-linear operation; it isolates the objects by converting the grey scale images into binary images. In this operation we select a threshold value and then each and every in the grey scale image is being compared with threshold level. If the pixels intensity is higher than threshold, the corresponding pixel is assigned with binary digit 1 or white in the output image. If the pixel intensity is less than the threshold level then that corresponding pixel is assigned with binary digit 0 or black in the output image. The threshold image with black and white is shown in figure 6.

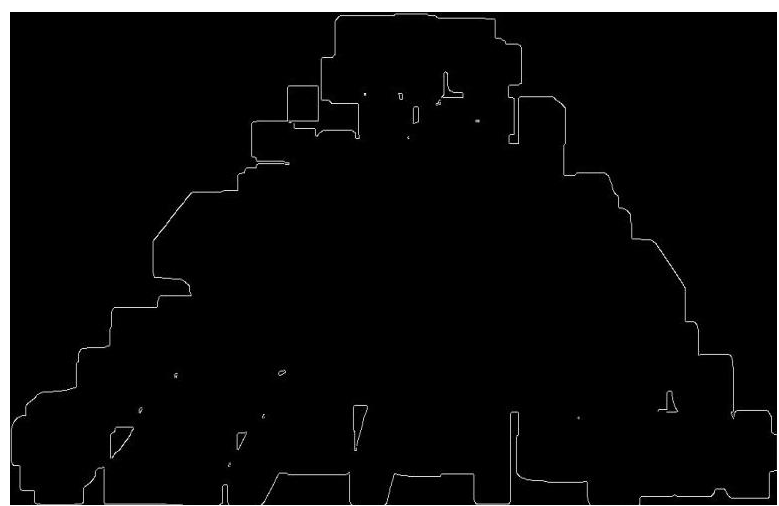

Fig.7. The canny edge detected traffic image

Then resultant image is processed by canny edge detection where the edges of traffic image is detected and removed other edges is shown in figure7. It also finds the intensity gradients of image. In the above figure we can see the edges of the traffic density.

Based on the intensity gradients and edges of the image, the micro controller compares all other road side traffic images and then it sends the commands to the traffic lights by adjusting timings for each green and red signal lights. This procedure is continuing throughout the day.

\section{CONCLUSION AND FUTURE SCOPE}

The developed system has minimized the occurrence of the traffic congestion. This work has been alleviated the work load on traffic cops, and reduced the waiting time of motorists. This project requires four high definition CCTV cameras hence it is cost effective and still the benefits of the system can be gained, it makes traffic flow more flexible.

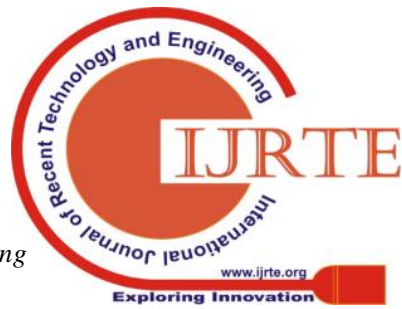


In future by designing efficient algorithm, the whole traffic and traffic lights in particular areas can be controlled by a centralized system.

\section{REFERENCES}

1. N. Kham, and C. Nwe, "Implementation of modern traffic light control system", International journal of scientific and research publication, vol4, issue 6, Jun. 2014.

2. International journal of Innovative research in science and engineering technology volume 3, March 2014 Density based traffic signals system by K. Vidya.

3. Motion sensors, CCTV's at signals to ease traffic in gurgaon www.m.timesofindia.com sep 9, 2018.

4. S.Jaiswal, T. Agarwal, A. singh and Lakshita, "Intelligent traffic control unit". International journal of electrical, electronics and computer engineering, vol, issue.2, pp. 66-72 Aug. 2013.

5. Jaywant kumble, Pratik Kothawade May 2013 "Traffic control system using Image processing" International journal emerging trends in electrical and electronics vol 3 , issue 3 .

6. "Density based traffic light controller using arduino" Muhammad aqib December23, 2018.

7. "Design and implementation of a density based traffic light controller with surveillance system" Y. N. Udoakah, and I. G. Okure. Vol 36, No.4 October 2017. 